\title{
HENOCH-SCHONLEIN PURPURA AND ASCARIASIS: A CASE REPORT
}

Agatha Siqueira Afonso1, Sarah Polyane Silva Veloso1, Caio Alexandre Zanoni1, Regiane Duque Minardi Neves1, Marlon Sousa Lopes1, Cristina Medeiros Ribeiro de Magalhães1, Maria Custódia Machado Ribeiro1, Marne Rodrigues Pereira Almeida1, Aline Garcia Islabão1, *

1.Hospital da Criança de Brasília José Alencar, Brasília (DF), Brazil.

${ }^{*}$ Corresponding author: aline.reumato@hotmail.com

\section{BACKGROUND}

Henoch-Schönlein purpura (HSP) is the most common vasculitis in childhood. The cause is unknown, but several triggering factors are previous such as toxic, infectious diseases or neoplastic conditions. Parasitosis as a trigger is rare. The diagnosis is based on palpable purpura with predominance in the lower limbs, diffuse abdominal pain, biopsy showing typical leukocytoclastic vasculitis or proliferative glomerulonephritis with IgA deposition, arthritis or arthralgia and renal involvement. Treatment consists of nonhormonal anti-inflammatory drugs, corticosteroid therapy and even immunosuppressants, when refractory to corticosteroid therapy.

\section{CASE REPORT}

R.S.S., 5 years old, presented fever, abdominal pain and vomiting. Abdominal ultrasound showed mesenteric lymph nodes and intestinal parasitosis, receiving treatment with nitazoxanide for three days. Four days after the onset of symptoms, the patient had fever and abdominal pain and evolved with pain on mobilization, edema, petechiae and purpura in the lower limbs, petechiae in the buttocks, erythematous-violaceous macules in the right palmar and plantar region, in addition to edema of hands, arthralgia in elbows, knees and ankles (Fig. 1). She also had hematuria and oliguria. The child had an episode of intestinal bleeding. No evidence of systemic arterial hypertension. The girl had platelet count within the reference value, preserved renal function, eosinophilia, normal antistreptolysin $\mathrm{O}$ antibody, erythrocyte sedimentation speed increased, microscopic hematuria, positive fecal occult blood test and positive EPF for Ascaris lumbricoides (Fig. 2). Ultrasound (Fig. 3) and abdominal computed tomography (CT) (Fig. 4) showed images compatible with parasitosis. Given the above, the child was diagnosed with HSP and ascariasis. Treatment was carried out with prednisone, omeprazole and albendazole. The patient returned 10 days after discharge due to the worsening of purpuric lesions in the lower limbs, in addition to pain, edema, local heat and the presence of bullous lesions in the left popliteal fossa (Fig. 5). She needed treatment with oxacillin, in addition to methylprednisolone. The patient showed significant improvement in his condition and continues to be weaned from corticotherapy.
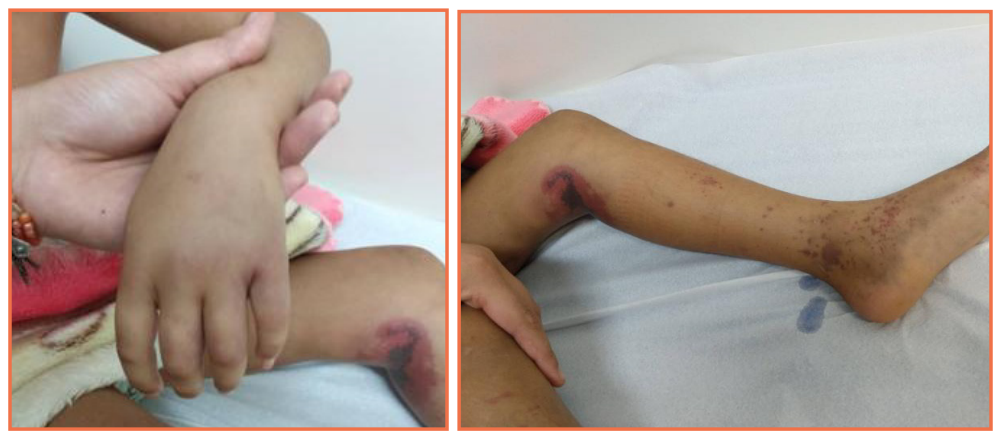

Figure 1. Hand edema and purpuric and petechial lesions in the lower limb.

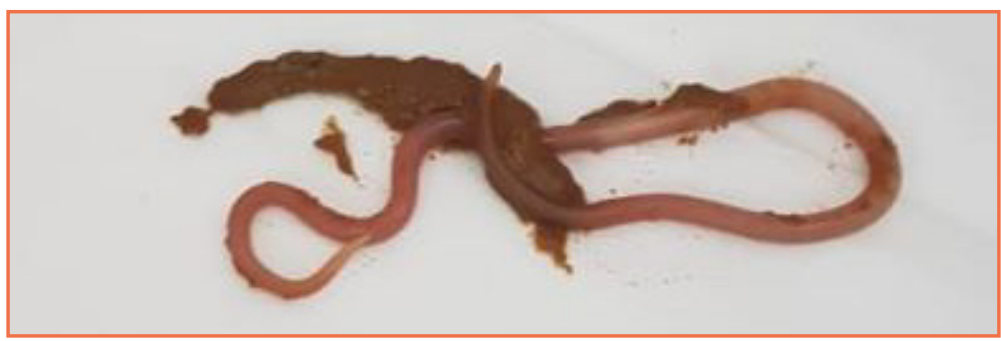

Figure 2. Elimination of Ascaris in feces. 

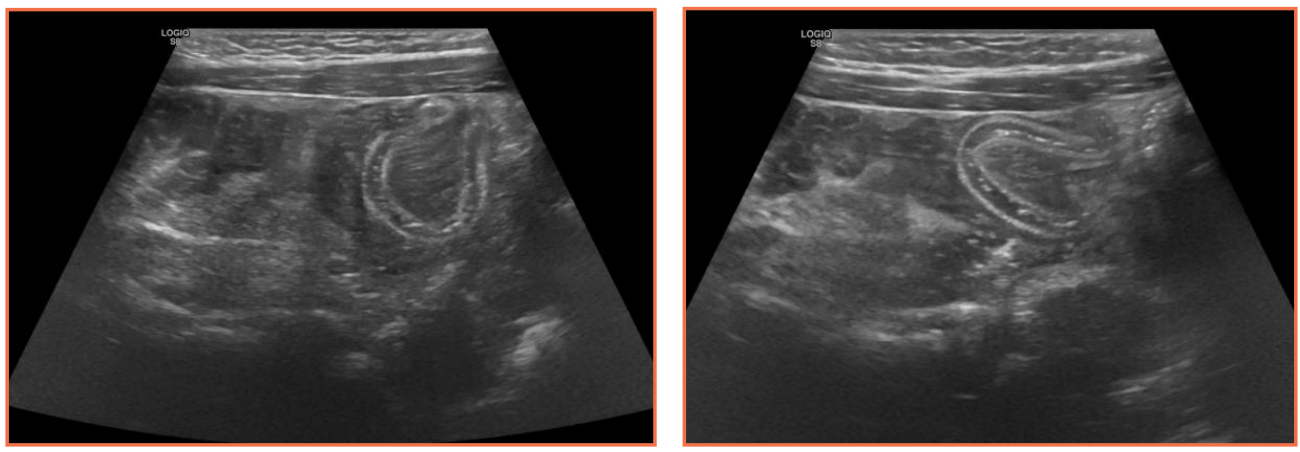

Figure 3. Abdominal ultrasound showing tubular images inside small intestinal loops.

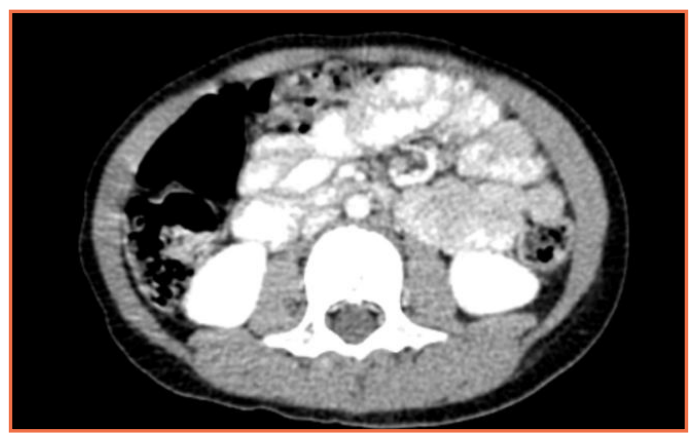

Figure 4. Abdominal CT with contrast showing a long tubular image in the ileal segment umen in the pelvic cavity, which also showed its light filling by positive contrast.

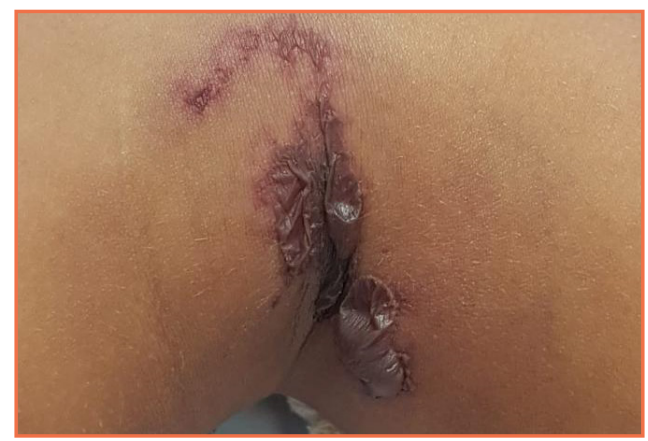

Figure 5. Purpura infected with bullous lesions in the left popliteal fossa.

\section{CONCLUSION}

There are not many records on ascariasis as a triggering factor for PHS; however, it is one of the most prevalent enteroparasitosis in humans and it affects mainly school children. In the girl in the case described, ascariasis was the trigger for the development of vasculitis, she received appropriate treatment and is being followed up by specialists. 